\title{
An Integrated Software Architecture for Rapid Product Development Processes
}

O. Eck, University of Stuttgart, Institute for Computer Science (IFI), Breitwiesenstr. 20-22 70565 Stuttgart, Germany, tel: +49 7117816 309, fax: +49711 7816-309, Oliver.Eck@informatik.uni-stuttgart.de

\section{W. Nogge}

Wolfram.Nogge@acm.org

D. Rantzau, University of Stuttgart, High Performance

Computing Center (RUS/HLRS), Allmandring 30, 70569

Stuttgart, Germany, tel: +497116855789, fax: +49711678 7626, rantzau@hlrs.de

M. Wolber, University of Stuttgart, Institute for Human Factors and Technology Management (IAT); Nobelstr. 12, 70569

Stuttgart, Germany, tel: +49 711970 2178, fax: +49 711970 2299, Mechthild.Wolber@iao.fhg.de

\begin{abstract}
In the complex process of product development, the ongoing distribution of processes and globalization of markets force companies to implement means of cooperation between partners at remote sites and coordination of tasks. In this paper we describe a distributed software architecture which provides an integrated set of tools for a wide range of Rapid Product Development processes. The central component is an active knowledge base called ASN (Active Semantic Network) which is used as shared data repository and team process coordination and upon which the other components of the system operate. The scenarios described are coming from the car manufacturing area ranging from collaborative development
\end{abstract}


of simulation prototypes using supercomputers to virtual reality analysis techniques.

\section{Keywords}

Rapid Product Development, Shared Design Databases, Collaborative Virtual Environments, Scientific Visualization, Information Visualization, Shared Understanding

\section{INTRODUCTION}

The question how development and testing of innovative products will manifest itself in practice is being investigated by interdisciplinary scientists at a Collaborative Research Center at the University of Stuttgart ${ }^{1}$. Taking an electrically adjustable car seat and the car interior as example, we intend to demonstrate that Rapid Prototyping techniques can be combined with an innovative structure of development teams and with improved cooperation and communication among scientists and managers to cut down development time and costs. The major goals of the Collaborative Research Center are the development of new processes in order to accelerate virtual and physical prototyping and to support scientists and managers by means of intelligent cooperation and communication systems. Autonomous expert teams will be provided with a common knowledge base, which can depict functional dependencies, quality characteristics, costs and manufacturing conditions as well as their interactions. Scientists from Industrial Psychology, Ergonomics, Business Management, Production Plants, Computer Science, Design Engineering, Synthetic Material Science, Laser Technology, Mechanical Engineering and Metal Forming cooperate in the Collaborative Research Center to reach these goals. According to the interdisciplinary structure, the Collaborative Research Center is divided into several areas. In the area described in this paper the computer scientists scope is to establish a team-oriented software architecture for Rapid Product Development processes.

\section{RAPID PRODUCT DEVELOPMENT}

The process of product development, we look at, is based on the concept of evolutionary product design (Rapid Product Development - RPD). Contrary to traditional concepts, RPD is characterized by short control loops within the process of product data generation and according management processes. The overall RPD approach is based on the idea of evolutionary design cycles. In contrast to traditional approaches with defined design phases and referring documents, e.g. specification list or concept matrix, different design cycles are carried out result-

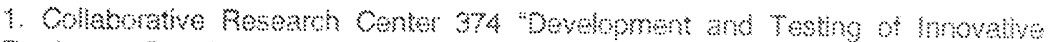

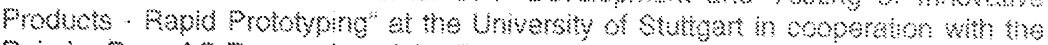

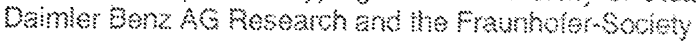


oriented. A design cycle starts basically with the phase plan \& conceive. During this segment initial concepts are selected and industrial design studies and planning issues (time, capacities, finances) are elaborated. In the following design phase, sub-systems and interfaces are analysed and defined, materials and tolerances determined as well as drawings elaborated (overall and part), which serve as a basis for the generation of a prototype. The prototyping phase ends with the generated prototype (physical or virtual) and the documentation of manufacturing or generation experience. Test protocols are written in the check segment, where tolerances, quality standards and lists of requirements serve as a basis. In the evaluating segment results are evaluated and analysed, which leads to a learning process. Results of each phase of the design cycles will be stored in the Active Semantic Net to support this learning process.

The whole RPD process is constrained by the project environment, such as market developments, legislation or new technologies. Furthermore the execution of cycles is not necessarily sequential, e.g. if results from the prototype generation lead to experiences they can be incorporated directly in a new design segment. The idea of evolutionary design implicates that new product requirements or technical progress must be taken into account and incorporated. This issue leads to an important feature of Rapid Product Development, namely the abandonment of a homogenous definition of the product modules within certain borders. The initial concept is made for the complete product as well as the final integration of the modules. In-between changes are done via new design methods and tools.

Communication and coordination as key factors are required in order to think in processes. The application of a process determines the product development and its effectivity and efficiency. One can distinguish between product data generation and management processes. Hence it is important to achieve a process orientation in the sense that both, product data generation and management processes, are aligned at the value chain. Innovation itself is achieved as a result of an initial product concept with the referring product specification in a traditional Simultaneous Engineering approach, whereas in RPD the concept will be checked and redefined (up to a certain stage) according to the project progress. RPD therefore offers the possibility to introduce new technologies, market trends (or even ideas) for a much longer development period leading to more innovative products. Design iterations are a wanted and therefore supported element in RPD. The change of design concepts and specifications is supported by a fitting framework, involving the test and most important the evaluation of the design for its further improvement.

\section{RPD - EXAMINED SCENARIOS}

Due to the possibility of the RPD process to incorporate new/changed product requirements, market trends and ideas or technical progress, the process itself is highly flexible and dynamic: teams, sub-teams and task forces of different experts can be established to solve specific problems in the course of the development process. 
In the following we present different prototyping processes, we examined within the development process. They occur at different phases of the development process: In the early phases of the development process we concentrate on the thermal comfort analysis in the car cabin. The following scenario gives us an insight in the shared understanding process of different experts like designer and manufacturer within the product development process to evaluate the manufacturing constraints within the common knowledge base.

\subsection{Simulation Prototype Scenario}

For car manufacturing, the early availability of virtual prototypes generated from large scale simulations can be crucial for the efficient development process, especially in the early phases, where physical prototypes are too cost intensive or simply to far away from being realizable in all its details. Those large amounts of scientific data is typically coming from crash or computational fluid dynamics simulations running on supercomputers in the pre-design phase in order to optimize certain properties such as the fuel consumption of a car engine or the thermal comfort in a car cabin. The interactive access to remotely connected supercomputer resources can dramatically shorten the iteration cycles of the various parameter studies usually performed during the whole simulation process.

With the increasing (global) distribution of development teams working on those "simulation prototypes", the large automotive companies today rely on an efficient telecommunication technology helping to keep the ongoing activities connected and synchronized. To keep the fulfilled tasks visible to other project members it is especially necessary to have a common knowledge base which is able to propagate changes in the design immediately to the appropriate knowledge sources, i.e. the responsible team members. In a phase where final design is a moving target, such a knowledge base serves as an active component communicating data and meta-data coming from several sources ranging from cost management, CAD construction to the simulation results and parameters mentioned. The typical phases involved in creating and optimizing a virtual simulation prototype where important design changes are triggered by the information gathered through the simulation process is shown in figure 1.

In the first phase, an initial CAD model is constructed or derived from existing models. Using this data, the grid for the fluid flow or FEM simulation can be built. Often the existing numerical model has to be altered and/or boundary conditions have to be initially defined. In the third phase the simulation, typically using large supercomputers, is performed. Here, several parameters (e.g. temperature, velocity,...) can be changed often triggering a re-calculation of the whole model. When simulation output data becomes available, post-processing steps such as filtering and mapping are applied to make certain properties of the model visible for the engineer. The data analysis is then followed by discussions (phase 5) with the responsible design/construction team members, when problems with the existing prototype have been encountered. In figure 1 the feedbackloops between the described phases are also shown. 


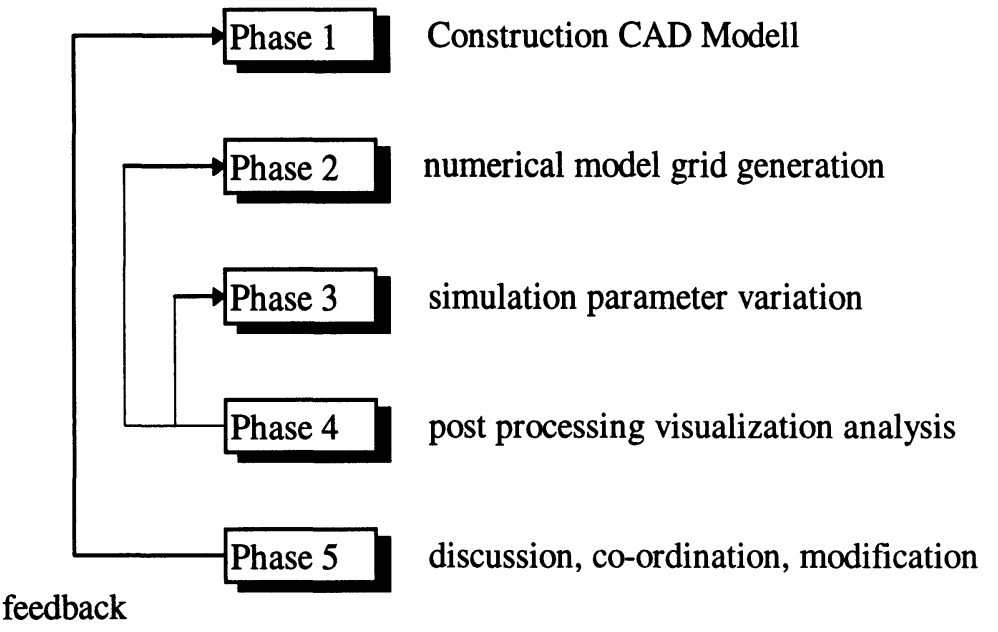

Figure 1: Phases in the simulation prototype scenario

The feedback between phase 4 and phase 3 means that the visualization is directly coupled to the running simulation i.e. allowing for interactive parameter steering. Going one step further, phase 4 can be directly coupled with phase 2 - the visualization/analysis can directly alter the numerical model e.g. modify the grid topology. It is often necessary to use CSCW techniques like audio/video conferencing for synchronizing the activities described: the simulation engineer has to talk to the designers and constructors often located at different places in the company. Moreover, when using high performance computing resources in phase 3 , the simulation people often have to talk to the providers of such resources, e.g. people at an in-house of externally located computing center maintaining the hardand software needed for this task. Ideally the construction people discuss the results found in the simulation directly using the visualization of the numerical model - the virtual prototype.

By using advanced virtual reality technology such as projection based virtual environments like the CAVE (Cruz-Neira, 1993), the abstract model can be explained much easier by the experts in the field to other project team members. Using adaptive and intuitive post-processing and visualization techniques, the bridge between numerics and design can be much more easily built. The results of phase 5 have to be distributed in several ways. The raw data can often only be placed on large file servers located near the supercomputers used. Meta-data about all the simulation tasks (parameters, boundary conditions, grid used) are transferred to the knowledge base including a link to the raw data. It is often at least desirable to have down-stripped 3D data available. Thus the construction engineers later can view and compare certain prototype using standard web-browsing tools (feedback from phase 5 to phase1). 


\subsection{Shared Understanding Scenario}

In future, it will be necessary to link up product development, exploration of knowledge and work in the laboratory more closely. This will, on the one hand, enable companies to optimize design and accuracy and, on the other hand, join the tasks of presentation and analysis within the engineering process much closer together. The common practice today is to base simulations on technological know-how, which means that if it is considered at all, the virtual environment is only taken into account at a later stage. Direct linking of the production process with virtual laboratories working on the aspects of design, ergonomics, hydro- or aerodynamics and prototyping, offers the advantage that construction know-how can be altered dynamically.

Virtual laboratories working on the aspects of design, ergonomics, hydro- or aerodynamics and prototyping help to illustrate looks and features of the product being designed. The generation of virtual prototypes, research in the mechanics of fluids as well as tests regarding ergonomics are well-known areas of application for virtual reality. The creation of virtual laboratories offers the possibility to generate various specialized views of one and the same product which is in the process of being designed, depending on the aspect to be emphasized. This means that all experts involved in the development process can refer to a common basis for cooperation.

One of the major challenges regarding modern product development is the practice of interlinking information from several special fields so that it can be used within all phases of the production process. In this context the know-how concerning process, construction and technology (e.g. quality/cost/time-analyses or simulations) plays a particularly significant role. It is however a fact that complex circumstances and networked know-how in combination with a large volume of data lead to an intricate stock of data. If this data is to be explored in an intuitive way, using visualisation and virtual reality can be the key. But there is still the problem of presenting this data in such a way, that it can be comprehended by humans, and that there are often no suitable mechanisms to interlink the information and thus enable interaction between the various experts.

Many complex tasks and problems are, as mentioned above, characterized by a large and three-dimensional stock of data. The creation of this stock (through measurements, evaluation, real-time simulations, scientific computing) involves enormous expenditure. Many effects that emerge in the field of mechanics of fluids, can only be registered by using techniques of visualisation. Of particular importance are, in this context, methods of presenting 3-dimensional as well as abstract stocks of data and the appropriate mechanisms for navigation.

\section{COMPONENTS OF THE INTEGRATED SOFTWARE ARCHITECTURE}

In order to fill the scenarios described above with life, we have developed a collaborative prototyping environment which allows visualization of complex 
geometry or simulation data, access to a shared knowledge base and adaptation of the different expert tools to provide a common understanding of the development process. The usage of these tools is tied together by an agent-based communication platform. The described overall scenario from the hardware point of view can be seen in figure 2.

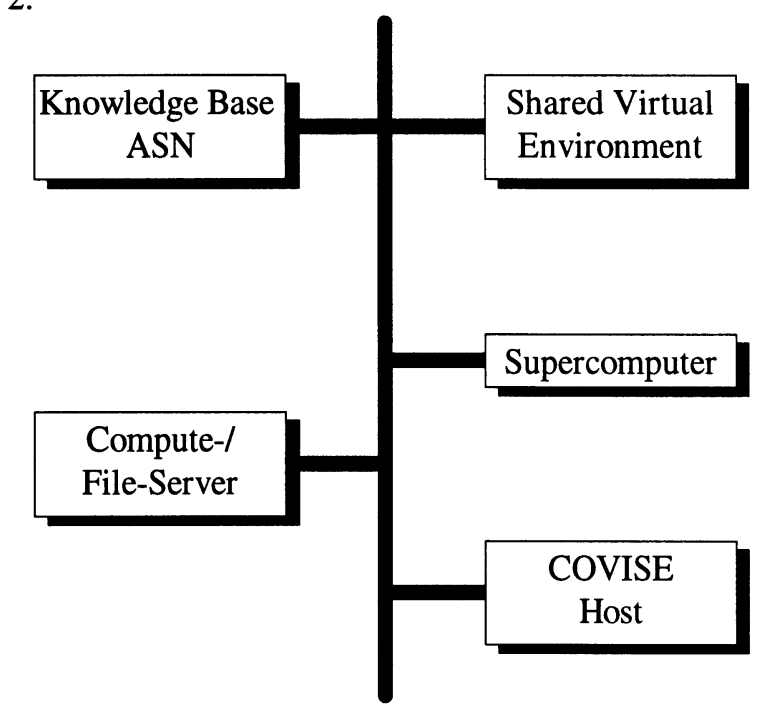

Figure 2: Components in a distributed simulation scenario

\subsection{Active Semantic Network}

The Active Semantic Network (ASN) is a knowledge base for representing all information relevant in the RPD process. The ASN represents information about all phases of this process (e.g. the product model and links to simulation and postprocessing results) and also includes information about the goals of the product design, e.g. the costs of the product, its quality and the design time. A survey of the ASN is given in (Roller, 1997A).

The ASN is an important component of the presented software architecture. As a shared repository, the ASN helps to combine different design results and serves as a communication medium for the designers. The ASN approach regards modeling as a basis for cooperative product development by developing a common understanding of the subjects of product design. Persons involved in product design typically have different expertise and the information stored in the knowledge base is therefore very interdisciplinary. In order to understand the meaning of product models defined by different persons, approaches to represent the semantics of database objects are realized in the ASN. Special modeling techniques and modern database concepts are used to represent this kind of information and different approaches are integrated to combine the different knowledge areas to a single product model. Default values and approaches to 
represent incomplete data models allow to adapt the knowledge base to the increasing amount of information resulting from the RPD process.

According to (Barghouti, 1991), traditional database concepts are too restrictive to fulfill requirements modern $\mathrm{CAD}$ environments place on product database systems. Therefore, the ASN integrates new concepts of object-oriented, distributed and active database management systems with a cooperative transaction model. Objectoriented database concepts are used in the ASN to represent complex design objects and inter-object dependencies. The cooperative transaction model provides a higher degree of parallelism and concurrency than traditional transaction models. Group-oriented mechanisms are integrated to allow cooperative accesses to shared database objects.

The ASN allows users to model constraints between data objects of different designers by dependency graphs (Roller, 1997B). Additional to an automatic propagation of constraint values, an automatic user notification is provided to inform designers about updates of other designers that are relevant for their work. Supporting communication and cooperative work inside of design teams helps designers to integrate partial models and to combine their ideas. Approaches of active database systems (McCarthy, 1989) are used to provide an active information delivery. The active behavior of the ASN is realized by ECA rules (Event-Condition-Action rules). These rules are used to model complex constraints and inter-object dependencies and to realize information flows and adaptive workflows inside design teams. The ASN also integrates information about the designers involved in the RPD process. A user model provides information necessary for the user interaction and group-oriented database accesses. The overall architecture of the ASN is presented in figure 3 which shows different partial models connected together by a dependency graph in the ASN knowledge base. 


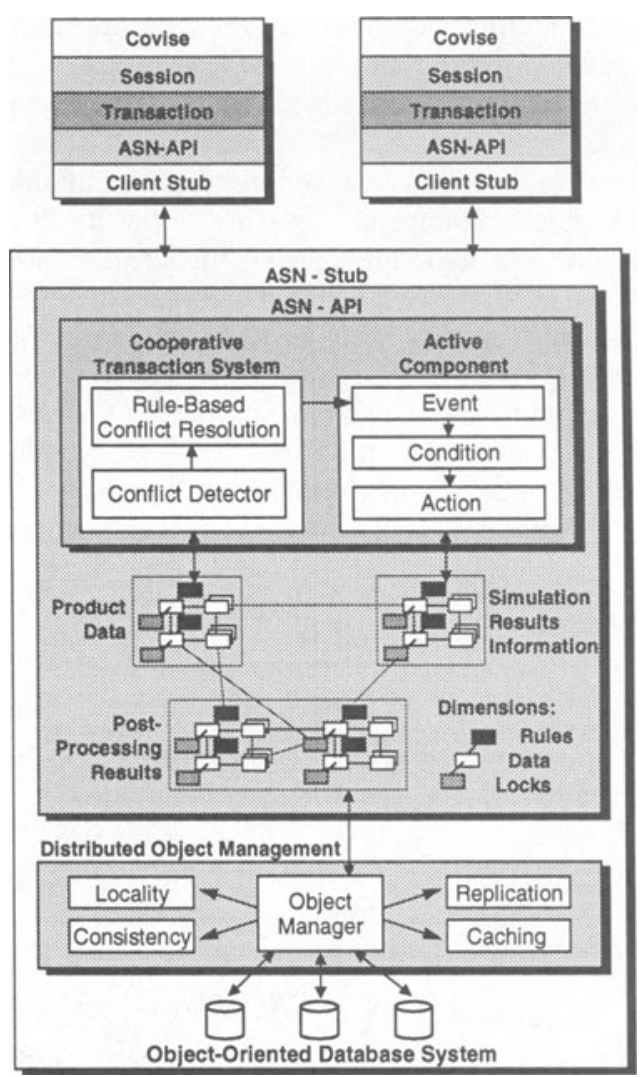

Figure 3: Architecture of the Active Semantic Network

The ASN is realized as a distributed database system in order to allow geometrically distributed design teams to access and manipulate shared data. Heterogeneous information sources are integrated by a Distributed Object Management (DOM) which provides users a transparent access to physically distributed data. This object management system is realized by a client/server architecture and an implementation of the Common Object Request Broker Architecture (CORBA) defined by the Object Management Group (OMG).

\subsection{COVISE}

COVISE is a software environment which integrates scientific visualization and simulation tasks across heterogeneous hardware platforms in a seamless manner. It has especially been optimized for efficient network transfer and high performance computing environments. The map editor user interface is based on the visual programming paradigm allowing to build distributed applications by combining modules (modeled as processes) from different application categories on different hosts to form more or less complex data-flow networks. At the end of such 
networks usually the rendering step does the final visualization of the numerical data. Session management and module synchronization is done in a central controller module which has the knowledge about the whole application topology. Request brokers on each participating host take care of data management and transfer. Modules on a single host use shared memory communication to minimize copying overhead. A special feature of COVISE is that it allows several users at distant sites to work in a collaborative way. All partners see the same screen representations at the same time on their local workstation in a shared application. The results of the visualization as well as user interactions are displayed in a synchronized way at each site. Standard audio/video conferencing facilities are used for discussion support. The scalable approach of COVISE allows single workstation desktop usage as well as multi-site networked collaboration including back projection based VR equipment (Rantzau, 1996), (Rantzau, 1998). A screen snaphot of a COVISE session involving three partners can be seen in plate 1:

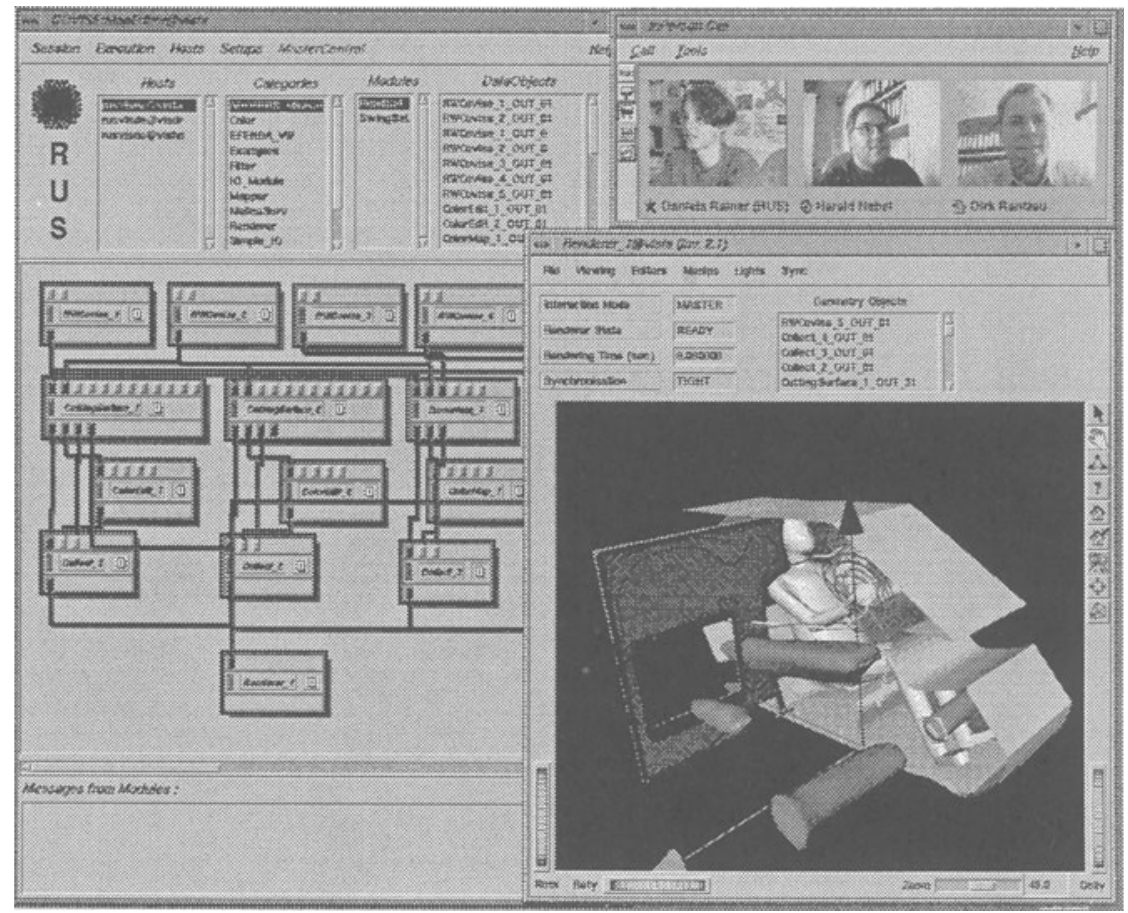

Plate 1: Analyzing the thermal comfort in a car cabin using the COVISE environment

Here, three engineers are analyzing the thermal comfort in a Daimler Benz car cabin. On the right side the rendering window showing the simulation results can be seen. On the left side the data-flow network combining the tasks like calculating temperature iso-surfaces or particle traces in the flow field is shown. COVISE has successfully been used in the described "Simulation Prototype Scenario" to support 
the phases 4 and 5 (Rantzau, 1997). The next step will be the integration of the phases 2 and 3 into the software environment including the ASN acting as knowledge exchange and repository. This will be part of the virtual laboratory which is described in the next section.

\subsection{Shared Understanding Environment}

First let's have a look at the specific topics which the application Shared Understanding Environment (SUE) is concerned with. SUE presents information and knowledge from different disciplines (construction, time-cost-quality management, aerodynamics, design) in an integrated environment. This means that SUE has to support the presentation of geometrical data (CAD, simulation, prototyping) as well as of abstract data (time-cost-quality, materials). In these various fields of application, there are many different kinds of presentation facilities, including, e.g. simple charts as well as volume visualisation. In order to achieve a domain-overlapping way of thought as well as to intensify the user's mental capacity, SUE should even be able to combine these presentation facilities. For this kind of integrated and adaptive information presentation, virtual reality (VR) represent a powerful visualisation and interaction facility.

Because of its high compute power, VR-technology enables us to create an integrated platform for adaptive views on interdisciplinary engineering problems. Therefore, SUE puts five principles into practice:

- We use spatial concepts to enable the understanding of knowledge derived from various different disciplines.

- We integrate virtual labs in order link up design, analysis and construction more closely.

- We try to combine different forms of presentation by using interieur/furniture.

- We have a variable degree and granularity of combination of knowledge spaces.

- We use metaphors in order to support user's cognition. 


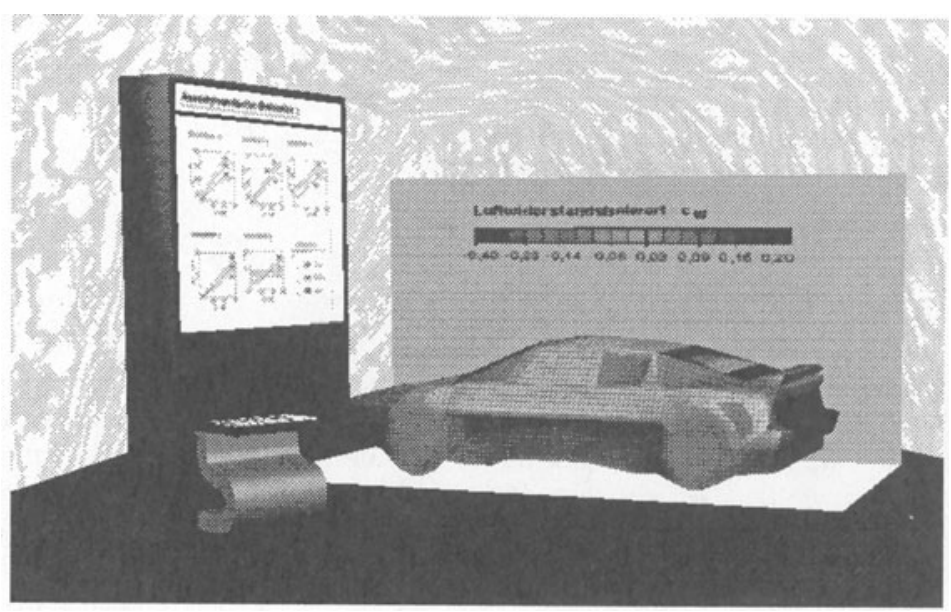

Plate 2: The interior of the aerodynamics laboratory

The aim of SUE is to optimize development processes through close inter-action of the individual disciplines. The close inter-linking of design, analysis and construction furthers an iterative, evolutionary approach to product development. This can shorten product development significantly, reduce costs and increase the quality of products. By implementing virtual laboratories (for construction, design, aerodynamics - see plate 2, ergonomics, materials) it becomes possible to create different specialized views onto one single object being designed. This tool can serve as an excellent basis for the work and cooperation of all experts involved in the development sector. The detachment from all traditional methods and approaches shall, in the following, be illustrated by positioning the work environment into outer space.

The resource "information", as a basis of all development and decision processes within a company, is gaining more and more importance. One precondition for fast and successful solution of development tasks is the efficient cooperation of experts from various fields. Virtual aerodynamics, design and ergonomics laboratories are helping the expert to imagine the appearance and features of the product being constructed. 


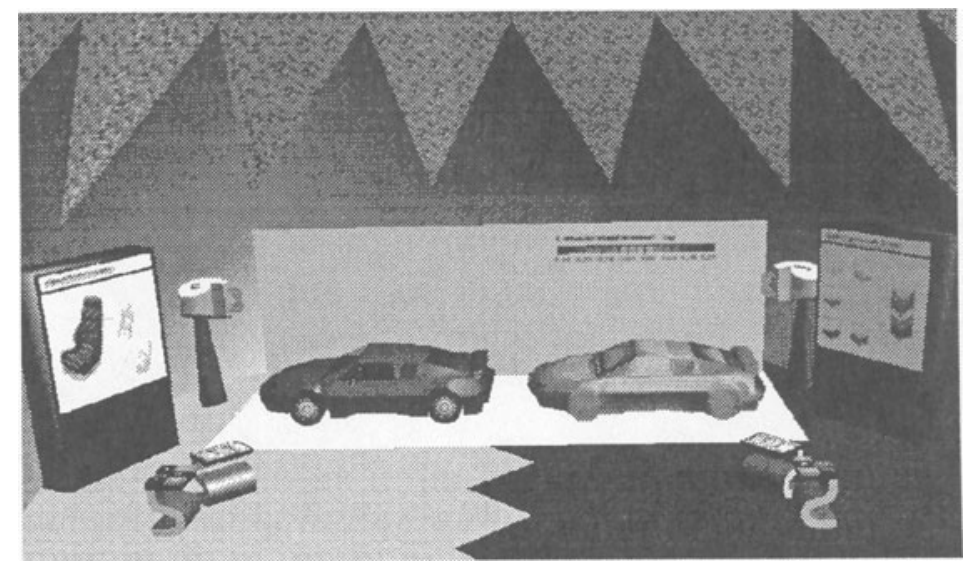

Plate 3: Combination of design and aerodynamics laboratory

Of similar importance is the fact that sources of knowledge about material data and supplier parts are integrated into the system. Our tools and methodologies offer the possibility to optimize design and accuracy in the engineering process by linking layout and analysis closely together, see the combination of design and aerodynamics laboratory in figure 6 . The laboratories inter-connect the various specialized departments from the organizational as well as from the informationtechnological point of view. Thus, SUE allows to explore large amounts of networked product and process information from various specific viewpoints taken by the different experts.

\subsection{Team-oriented communication system for cooperative work}

In the RPD process, experts from various departments have to come together in a team: designers, engineers, production and assembly specialists and controllers are all experts in their field, and their individual knowledge and know-how is therefore important for the development process. The development process as such is, however, not clearly predictable. Questions, problems, etc. have to be solved, the advice of experts have to be sought and interfaces with components have to be redefined and specified. Often teams and sub-teams are employed to organize these tasks and fulfil the corresponding requirements. This requires a great amount of communication and coordination among teams, which is most time consuming.

In principle, computer-supported tools (CSCW systems) can be employed to support these teams and groups in order to facilitate and improve communication processes and the coordination of tasks. Synchronous CSCW tools as audio/video conference systems, joined editing or shared applications make it possible for several users to work simultaneously on the same task, by distributing the ad-hoc communication to several screens. Asynchronous CSCW tools support a working technique were the members of staff work on the task at different times. Some of the better known tools in this category are for example e-mail and work flow management systems. These tools are, however, only partially suited for the use in 
product development. Simple e-mail systems neither embody any semantic structure on which their messages are backgrounded nor do they allow the user to track the stages of a project. Work flow management systems have the great disadvantage that, in order to be used effectively and efficiently, they require a known, structured process. But unfortunately this type of process very rarely exists in the field of development, as one of the characteristics of this field is that it involves rather unstructured, unpredictable processes.

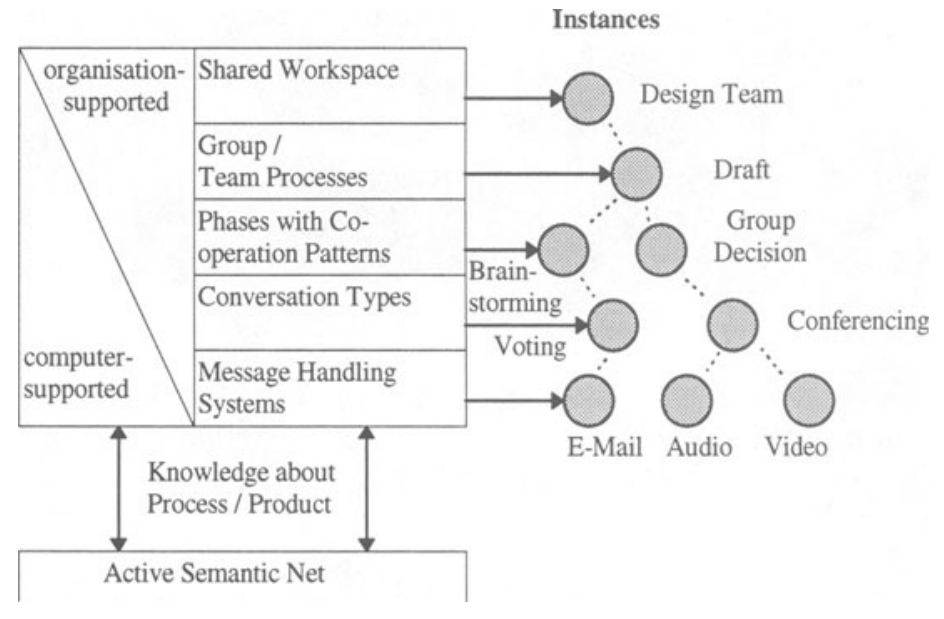

Figure 4: Hierarchical model for Shared Workspaces

To intensify team coordination during the phase of development, so called shared workspaces seem to be a promising means. Shared workspaces coordinate the flow of information between individual members of the team in an associative way. They provide materials, e.g. information objects, and tools for manipulation, which serve to reach the common objectives of a team. To cope with the complex information and coordination infrastructure, we built a hierarchical model for a shared workspace. It is formally defined by the following five layers: Shared Workspace, Group and Team Processes, Phases with Cooperation Pattern, Conversation Types and Message Handling System. The lower layers are more technic-oriented, whereas the upper layers are more are affected by organizational concerns. The different layers are described in the following (figure 4).

Shared Workspace: A shared workspace is used to support different experts concerned with the cooperation and aiming to fulfill a common task. Thus the aim is to enable these persons to collaborate as if they were in the same room. Therefore a shared workspace should make the respective working materials, (objectives of the cooperation) and tools (like CAD Modeling systems, FEM analyzer programs, spreadsheets etc.) available. With regard to organizational needs, the following components must be defined: members of the workspace (and their roles), working materials, filing structures and tools, which are used by the members for collaboration. 
Team Processes: Within the shared workspace, team processes are performed. Cooperation between team members within the RPD process is accompanied by alternating synchronic as well as asynchronic tasks. Sub-teams are built to solve partial problems. The various tasks and the course of events within such a team can be defined as a team process. A team process is defined as the specification of information, activities and attributes of an electronically supported team. A frame for the team tasks is defined. Team processes consist of specifications in the following areas: team organization, team protocol, team environment, group sessions, goal, activities, documents and state of the team. Team processes may consist of several phases. Each of these phases has a regular cooperation pattern.

Cooperation Pattern and Conversation Types: Phases within the team process are subject to a certain type of cooperation pattern (e.g. brainstorming, evaluation of ideas, decision support,...). They are specified by a conversation type. A conversation type consists of message types, a conversation protocol and a set of actors involved. With regard to the speech-act model, (Winograd, 1986) distinguishs five different categories of message types: assertive, directive, commissive, declarative and expressive. The contents of a message specify the item of the communication (topic). Each conversation type has precisely one conversation protocol, which is defined by a set of rules. Petri Nets (state-transition diagrams) or Role-Interaction Networks can be used to determine the rules of conversation. They define which message type can be followed by which other message type. The actors involved are either natural persons or software agents, who help individuals to perform their tasks. The state of actors can be changed depending on messages sent or received.

Message Handling Systems: Message handling systems are e.g. normal e-mail systems (POP-3, SMTP, IMAP4) or distributed information systems (like the world-wide web) or centralized databases. Our objective is to integrate the shared workspace into the Active Semantic Net in order to support the RPD process.

\section{CONCLUSION}

In this paper we introduced a flexible and dynamic system layout for the integration of shared workspaces into the RPD process. The various experts can be involved in several workspaces. Based on our model of shared workspaces we are currently implementing a system for adaptive workflows. As message handling system we are using the Active Semantic Network, which contains all information about process, product data and users being involved. Our next objective is the entire integration of this version into the Active Semantic Network and a construction tool. Furthermore we have to extend this model to synchronic uses (like shared-application, whiteboard, audio-video) and events.

\section{REFERENCES}

Abbott, K.R. and Sarin, S.K. (1994) Experiences with Workflow Management: Issues for the Next Generation, in Transcending Boundaries; Proceedings of 
the Conference on Computer Supported Cooperative Work (ed. R. Furuta), Chapel Hill, NC, USA. New York, NY: ACM, 113-20.

Barghouti, N.S. and Kaiser, G.E. (1991) Concurrency Control in Advanced Database Applications. ACM Computing Survey, Vol. 23, No. 3, 267-317.

Borghoff, U.M and Schlichter, J.H. (1995) Rechnergestützte Gruppenarbeit. Berlin; Heidelberg et al.: Springer-Verlag.

Brown, J.R.; Earnshaw, R.; Jern, M.; Vince, J. (1995) Visualization - Using Computer Graphics to Explore Data and Present Information. John Wiley \& Sons, Inc.

Card, S.; Eick, S.; Gershon, N. (1996) Proceedings IEEE Symposium on Information Visualization '96, IEEE Computer Society Press.

Cruz-Neira, C.; Sandin, D.J.; De Fanti, T.A. (1993) Surrround-screen projectionbased virtual reality: design and implementation of the CAVE, in: $A C M$ SIGGRAPH '93 Proceedings, 135-42.

Dourish, P.; Holmes, J.; MacLean, A.; Marqvardsen, P.; Zbyslaw, A.: Freeflow (1996) Mediating Between representation and Action in Workflow Systems, in Proceedings of the ACM 1996 Conference on Computer Supported Cooperative Work (ed. M.S. Ackerman), New York: Association for Computing Machinery, Inc., 190-8.

Gallagher, G.S. (1995), Computer Visualization: Graphics Techniques for Scientific and Engineering Analysis, CRC Press.

McCarthy and Dayal, U. (1989) The Architecture of An Active Data Base Management System, in Proceedings of ACM SIGMOD International Conference on Management of Data, 215-24.

Pankoke-Babatz, U. and Syri, A. (1996) Gemeinsame Arbeitsbereiche: Eine neue Form der Telekooperation? In Herausforderung Telekooperation, Fachtagung Deutsche Computer-Supported Cooperative Work 1996, (eds. H. Krcmar; H. Lewe; G. Schwabe), DCSCW“96. Berlin, et al.: Springer-Verlag, 51-67.

Rantzau, D. and Lang, U. et al (1996) Collaborative and Interactive Visualization in a Distributed High Performance Software Environment, in High Performance Computing for Computer Graphics and Visualization (eds. M. Chen; P. Townsend; J.A. Vince), Springer, 207-16.

Rantzau, D; Lang, U. (Nov. 1997) A Scalable Virtual Environment for Large Scale Scientific Data Analysis, in Proceedings of the Euro-VR Mini Conference '97, Amsterdam, to appear in Future Generation Computer Systems, Elsevier, 1998.

Rantzau, D.; Ruehle, R. (1997) Analyzing the Thermal Comfort in a Car Cabin Using a Modular Virtual Prototyping Environment, in Proceedings of the 30th ISATA Conference, Florence.

Roller, D.; Bihler, M.; Eck, O. (1997A) ASN: Active, Distributed Knowledge Base for Rapid Prototyping, in Proceedings of 30th ISATA, Volume Rapid Prototyping in the Automotive Industries. Automotive Automation Ltd (ed. D. Roller), Croydon, England, 253-62.

Rosenblum, L.; Earnshaw, R. A.; Encarnacao, J.; Hagen, H.; Kaufman, A.; Klimenko, S.; Nielson, G.; Post, F.; Thalmann, D. (1994) Scientific Visualization - Advances and Challenges, Academic Press. 
Roller, D. and Eck, O. (1997B) Constraint Propagation Using an Active Semantic Network, in Geometric Constraint Solving \& Application (eds. B. Brüderlin, D. Roller), Springer-Verlag, 43-57.

VRML2 Language Specification, URL: http://www.vrml.org/

Winograd, T.and Flores, F. (1986) Understanding Computers and Cognition - A New Foundation for Design. Norwood, NJ: Ablex Publishing Corporation.

\section{BIOGRAPHY}

Oliver Eck studied Computer Science at the University of Stuttgart, Germany. After receiving his diploma in 1994, he worked as a Research Assistant at the Research Institute for Applied Knowledge Processing (FAW Ulm) in Ulm, Germany. Since 1996, he is working on his doctoral thesis at the Institute of Computer Science, University of Stuttgart, Germany. His interests focus on advanced concepts of design databases and product modelling.

Wolfram Nogge joined the Collaborative Research Center from January 96 to March 98. Currently, he is working for SAP in Walldorf, Germany.

Dirk Rantzau received the Diploma degree in Mechanical Engineering from the University of Stuttgart in 1993. Since 1995 he is working in the Collaborative Research Center in the field of VR based virtual prototyping. His current research interests include collaborative virtual environments for scientific visualization, 3D user interfaces and interaction techniques for computational steering.

Mechthild Wolber studied computer science in Karlsruhe, Germany. In 1995, after receiving her diploma, she joined the IAT, University of Stuttgart and the Collaborative Research Center, where she is responsible for the design of adaptive user interfaces. Her special research interests are work design, information visualization and information management. 